\title{
Performance Evaluation of AOMDV \& MPOLSR Routing Protocol for MANET
}

\author{
Rohit Jain ${ }^{1}$, Sourabh Jain ${ }^{2}$, Mukesh Patidar ${ }^{3}$, Piyush Agrawal ${ }^{4}$ \\ Lecturer ECE, Department of ECE, MIT, Mandsaur ${ }^{1,2,3,4}$
}

\begin{abstract}
A mobile ad hoc network (MANET) is a collection of wireless mobile nodes communicating with each other using multi-hop wireless Links without any existing network infrastructure or centralized administration. In recent years, a variety of routing protocols targeted specifically at this environment have been developed and some performance simulations are made. Multi-path routing has been studied thoroughly in the context of wired networks. It has been shown that using multiple paths to route messages between any source-destination pair of nodes (instead of using a single path) balances the load more evenly throughout the network. The common belief is that the same is true for ad hoc networks, i.e., multi-path routing balances the load significantly better than single-path routing. Our Protocol, called MP-OLSR \& AOMDV is a multipath routing protocol. In addition route recovery \& loop detection are implemented in MP-OLSR in order to improve quality of service regarding OLSR.MP-OLSR is suitable for mobile, large \& dense network with large traffic \& could satisfy critical multimedia applications with high on time constraints.
\end{abstract}

IndexTerms: MANET, MRP, AOMDV, MPOLSR etc.

\section{INTRODUCTION}

In MANETS, the main purpose of the convention or standard protocols is to control the way in which the mobile nodes decide how to transfer the route packets to each other. These protocols are broadly classified into three main categories namely proactive, reactive and hybrid protocols. Proactive protocols maintain routes to all nodes, including nodes to which no packets are sent. Proactive protocols include DSDV, OLSR, MPOLSR and WRP. In reactive protocols, routes between hosts are determined only when they are explicitly needed to forward packets. Reactive

Protocols include AOMDV, AODV, DSR, TORA and CBRP. Hybrid methods combine proactive and reactive methods to find efficient routes, without much control overhead. Mobile Ad hoc Networks (MANETs) are a class of wireless communication networks without a fixed infrastructure. The MANET concept has basically evolved to tackle the disaster situations like tsunami, earthquake, terrorist activities, battlefields, land-slides, etc. Later, the concept has been extended to include applications such as online education, gaming, business, etc. Several applications in MANETs need group communication to manage the situations. The MANET nodes do not provide reliable services and QoS (Quality of Service) guarantees as compared to other wireless networks such as Wi-Fi, WiMAX, GSM and CDMA. The main sources of unreliability in MANETs are due to limited battery capacity, limited memory and processing power, varying channel conditions, less stability under unpredictable and high mobility of nodes. The Qos parameters to be guaranteed for multimedia group communication are bandwidth, delay, packet loss, jitters and bandwidth- delay product. The measure of unreliability increases when we need to communicate real-time multimedia traffic where stringent Quality of Service (QoS) parameters are to be satisfied. QoS is one of the significant components to evaluate MANET performance since QoS restricts the bounds on bandwidth, delay, bandwidth delay product, and jitter and packet loss. The violation of these parameters degrades the overall performance of an application. Reliable multicast routing includes the mechanisms such as error detection, signaling of error messages to source and destination and retransmission method of lost packets [2]. Also, there is a existence of another approach where mobile nodes utilizing each other as access points or relays for traffic when they cannot establish a direct communication with end points. That model of communication is called mobile Adhoc networks (MANETS). These networks can be set up randomly and when needed (on demand). In MANETS nodes have to announce their presence periodically and listen for their neighbor's announcements broadcasts to discover and learn how to reach each other. Therefore, mobility and scalability are the main challenges in the infrastructure less networks. Hence there is need for efficient routing protocols to allow the nodes to communicate over multihop paths, consisting of several links, dynamic and non Predictable topology in a way that doesn't use any more of the network resources then necessary. Routing protocols for MANETs can be categorized in various ways. They can be classified as proactive and reactive routing depending on several factors. Such factors can be for example the time taken for routes discovery or routing information update mechanism. In proactive routing, every host maintains at least one routing table to represent the whole topology of the network. The tables (of each host) are updated continuously. Therefore, routes are already available at any time some hosts want to communicate with each other. In order to maintain up-to-date routing information at all hosts, topology information has tube exchanged between all hosts on a regular basis. This increases the overhead in the network. On one hand, substantial bandwidth is used for the large control traffic; 
on the other hand, routes are always available in shortly for any communication request. This reduces the delays of data transmissions. One of the most important proactive protocols is the Optimized Link State Routing protocol (OLSR) unlike proactive routing protocols; reactive routing protocols initiate a route discovery process when needed. This reduces the overhead as compared to proactive routing protocols, but it increases the transmission delay. Another classification can be made according to number of paths a routing protocol delivers per source destination pair [7]. There exist unipath and multipath routing protocols. Unipath routing protocol: one route is used to deliver data from source node to destination node. Multipath routing protocol: more than one route is used to deliver the data.

\section{MULTIPATH ROUTING IN ADHOC NETWORKS}

Mobile ad hoc networks (MANETs) are characterized by a dynamic topology, limited channel bandwidth and limited power at the nodes. Because of these characteristics, paths connecting source nodes with destinations may be very unstable and go down at any time, making communication over ad hoc networks difficult. On the other hand, since all nodes in an ad hoc network can be connected dynamically in an arbitrary manner, it is usually possible to establish more than one path between a source and a destination. When this property of ad hoc networks is used in the routing process, we speak of multipath routing. In most cases (e.g.), the ability of creating multiple routes from a source to a destination is used to provide a backup route. When the primary route fails to deliver the packets in some way, the backup is used. This provides a better fault tolerance in the sense of faster and efficient recovery from route failures. Multiple paths can also provide load balancing and route failure protection by distributing traffic among a set of disjoint paths. Paths can be disjoint in two ways: (a) link-disjoint and (b) node-disjoint. Nodedisjoint paths do not have any nodes in common, except the source and destination, hence the do not have any links in common. Link-disjoint paths, in contrast, do not have any links in common. They may, however, have one or more common nodes [5].

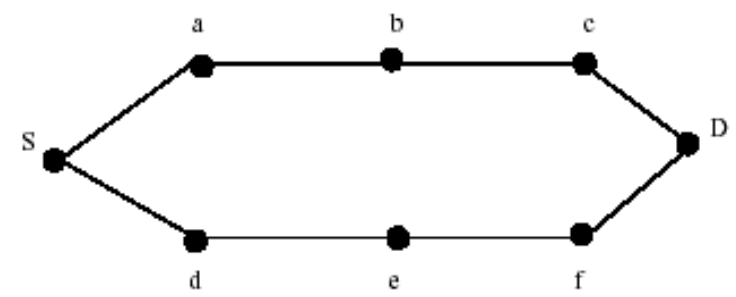

Fig 1. Two node-disjoint paths from source $S$ to destination $D$.

Fig 2.Two link-disjoint paths from source $S$ to destination $D$. Note that they are not node-disjoint, since they share node $b$.
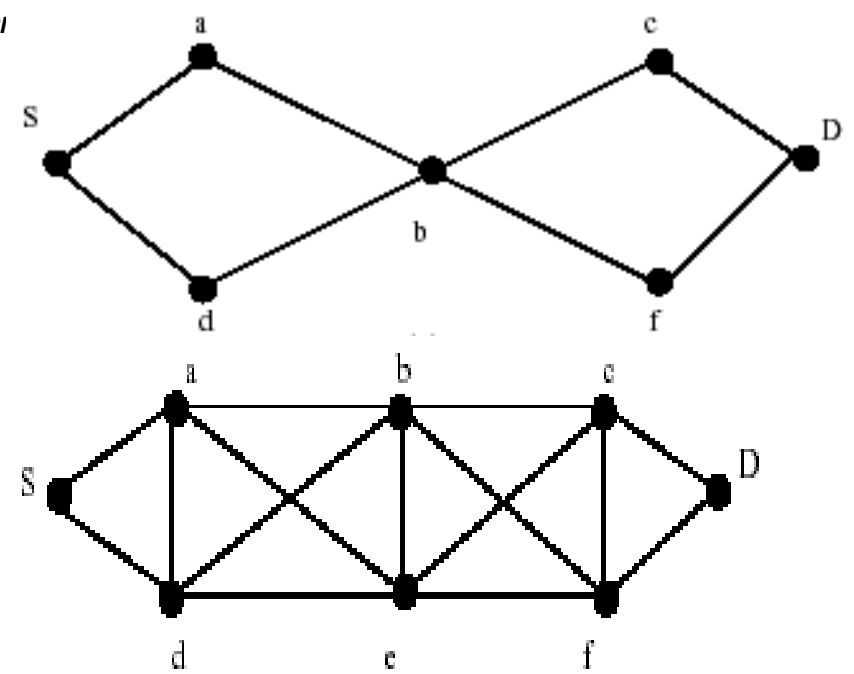

Fig 3.The two node-disjoint paths, when they are in each other's radio coverage.

In order to use multiple paths simultaneously they need to be as independent as possible. So not only do they need to be disjoint, also route coupling must be taken into account, because routes can interfere with each other. Route coupling takes place when a path crosses the radio coverage area of another path. There is a protocol that uses this property of radio broad cast to create backup-routes, but in the case of multiple-path data transport route coupling is unwanted. Routes may be link- or even nodedisjoint but still interfere with each other due to route coupling. Consider the node-disjoint routes of figure 1again. In the situation of figure 3 , when node $a$ for example sends data to node $\mathrm{b}$ (both route 1), node $d$ on the other route cannot transmit data to $e$ on route 2 , since the nodes (and thus routes) are in each other's radio coverage area and interfere with each other. Since none of the routing protocols take the route coupling into account, we will ignore it in the sequel. Disjointness will be the only measure used for path independence.

\section{AOMDV}

Ad-hoc On Demand Multipath Distance Vector Routing Algorithm (AOMDV) is proposed in [5]. AOMDV employs the"Multiple Loop-Free and Link-Disjoint Path" technique. In AOMDV only disjoint nodes are considered in all the paths, thereby achieving path disjointness. For route discovery route request packets are propagated throughout the network thereby establishing multiple paths at destination node and at the intermediate nodes. Multiples Loop-Free paths are achieved using the advertised hop count method at each node. This advertised hop count is required to be maintained at each node in the route table entry. The route entry table at each node also contains a list of next hop along with the corresponding hop counts. Every node maintains an advertised hop count for the destination. Advertised hop count can be defined as the "maximum hop count for all the paths". Route advertisements of the destination are sent using this hop count. An alternate path to the destination is accepted by a 
node if the hop count is less than the advertised hop count Packet Delivery Fraction (PDF): The ratio between the for the destination [8].

\section{MPOLSR}

The MP-OLSR can be regarded as a kind of hybrid multipath Routing protocol which combines the proactive and periodically to detect the network topology, just like OLSR. However, MP-OLSR does not always keep a routing table. It only computes the multiple routes when data packets need to be sent out. The core functionality of MP-OLSR has two parts: topology sensing and route computation. The topology sensing is to make the nodes aware of the topology information of the network. This part benefits from MPRs like OLSR. The route computation uses the Multipath Dijkstra Algorithm to calculate the multipath based on the information Obtained from the topology sensing. The source route (all the hops from the source to the destination) is saved in the header of the data packets. The topology sensing and route computation make it possible to find multiple paths from source to destination. In the specification of the algorithm, the paths will be available and loop-free. However, in practice, the situation will be much more complicated due to the change of the topology and the instability of the wireless medium. So route recovery and loop detection are also proposed as auxiliary functionalities to improve the performance of the protocol[9]. The route recovery can effectively reduce the packet loss, and the loop detection can be used to avoid potential loop sin the network as depicted in we discuss both the core functionalities and auxiliary functionalities[6].

\section{METHODOLOGY}

3.1 Simulation Environment

Simulation environment is as follows:

Parameter Values

\begin{tabular}{|l|l|}
\hline Traffic type & CBR \\
\hline Simulation time & 100 seconds \\
\hline Number of nodes & $25,50,75,100$ \\
\hline Pause time & $0,100,200,300,400,500,600$ \\
\hline $\begin{array}{l}\text { Maximum } \\
\text { connections }\end{array}$ & $5,10,15,20$ \\
\hline $\begin{array}{l}\text { Maximum speed of } \\
\text { nodes(m/s) }\end{array}$ & $5,10,15,20$ \\
\hline $\begin{array}{l}\text { Area of the } \\
\text { network }\end{array}$ & $1000 * 1000$ \\
\hline
\end{tabular}

\section{NS-2 (Network Simulator-2)}

The NS-2 [3] is a discrete event driven simulation and in this the physical activities are translated to events. Events in this are queued and processed in the order of their scheduled Occurrences. The functions of a Network Simulator [9] are to create the event scheduler, to create a network, for computing routes, to create connections, to create traffic. It is also useful for inserting errors and tracing can be done with it. Tracing packets on all links by the function trace-all and tracing packets on allinks in nam format using the function namtrace-all.

\section{Performance Metrics}

We report two performance metrics for the protocols: number of data packets received and the number of packets sent.

End-to-End Delay: It is the ratio of time difference between every CBR packet sent and received to the total time difference over the total number of CBR packets received.

\section{SIMULATION RESULTS AND ANALYSIS}

We ran the simulation environments for $600 \mathrm{sec}$ for four scenarios with nodes varying from 25 to 100 , pause times varying from 0 to 600 second, maximum connections varying in between 5 to 20 connections \& maximum speed varying in between 5 to 20 meter per second. Packet delivery fraction \& end to end delay are calculated for AOMDV and MPOLSR. The results are analyzed below with their corresponding graphs.

Packet Delivery Fraction

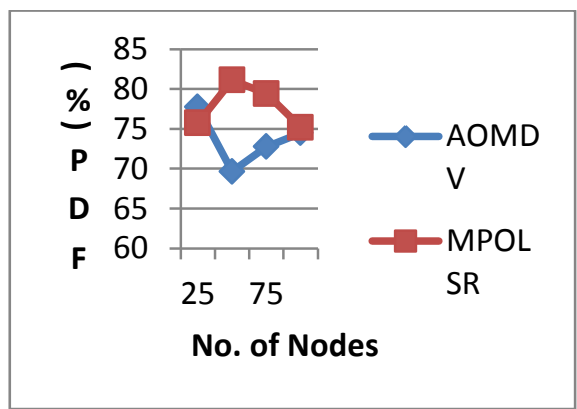

Fig: Comparison of AOMDV \& MPOLSR on the basis of PDF at Max.connection-10, Max.speed-20, pause time-0 \& by varying nodes.

We note that in this scenario at node 25 AOMDV performances is better, but as we are increasing the no of nodes MPOLSR performs well as compared to AOMDV

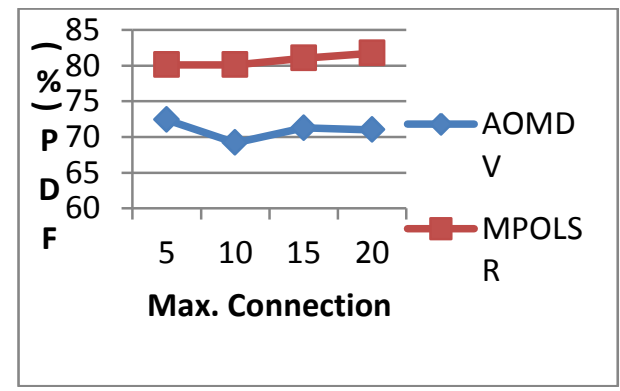

Fig: Comparison of AOMDV \& MPOLSR on the basis of PDF at Nodes-50, Max.speed-20, pause time-0 \& by varying Max.Connection

We note that in this scenario at connection 5 MPOLSR performance is better, but as we are increasing the no of connections MPOLSR performs well as compared to AOMDV. 


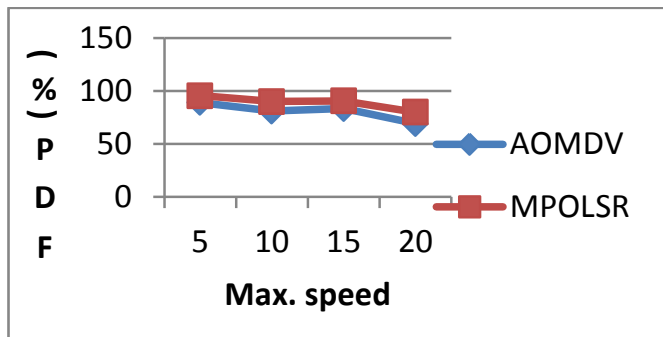

Fig: Comparison of AOMDV \& MPOLSR on the basis of PDF at nodes-50, Max.connection-10, pause time-0 \& by varying Max. speed.

We note that in this scenario at speed $5 \mathrm{~m} / \mathrm{s}$ MPOLSR performance is better, but as we are increasing the speed MPOLSR performs well as in all the cases as compared to AOMDV.

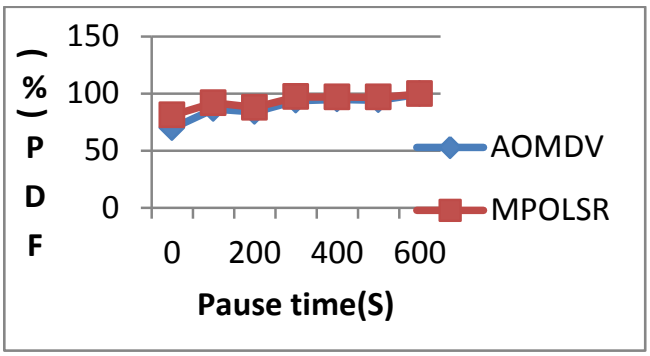

Fig:Comparison of AOMDV \& MPOLSR on the basis of PDF at nodes-50, Max.connection-10, Max.speed-20 \& by varying Pause time.

We note that in this scenario at pause time $0,100,200$ MPOLSR performances is better, but as we are more increasing the pause time MPOLSR performs some similar results as compared to AOMDV.

\section{ANALYSIS OF THE RESULT}

We ran the simulation environments for $600 \mathrm{sec}$ for four scenarios with nodes varying from 25 to 100 , pause times varying from 0 to 600 second, maximum connections varying in between 5 to 20 connections, maximum speed varying in between 5 to 20 meter per second and field area is of dimensions $1000 * 1000$.we note that in all the cases of PDF as we are comparing in our scenario MPOLSR performs well in all the cases but at one or two fixed value AOMDV performance is better and also showing some similar results by varying pause time.

\section{End to End Delay:}

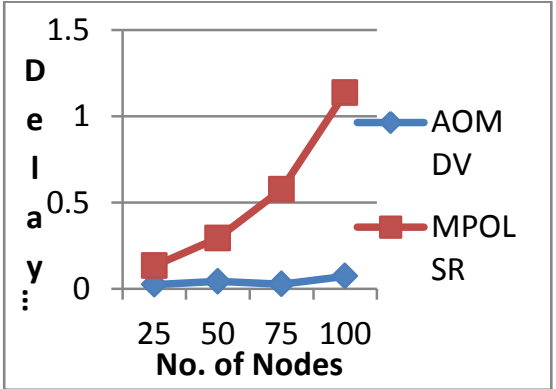

Fig:Comparison of AOMDV \& MPOLSR on the basis of delay at max. Connection-10, max.speed-20, pausetime-0 $\&$ by varying nodes.

We note that in this scenario delay in AOMDV \& MPOLSR is increasing by varying the no of nodes. But as we are increasing the no of nodes MPOLSR performs more delay as compared to AOMDV.

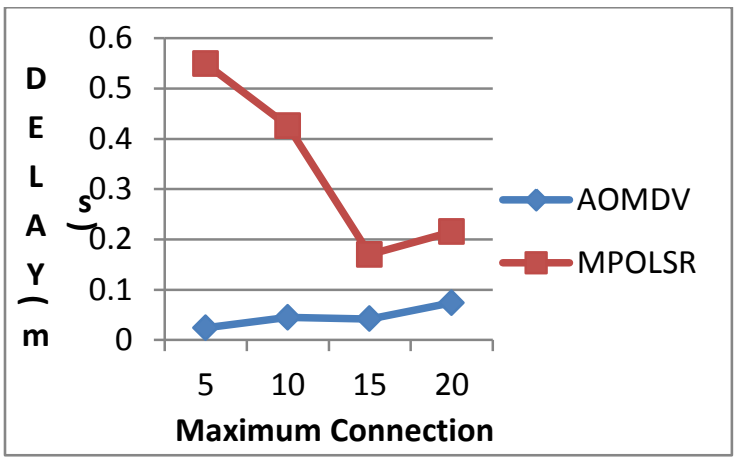

Fig:Comparison of AOMDV \& MPOLSR on the basis of delay at nodes-50, max.speed-20, pausetime- 0 \& by varying max. Connection.

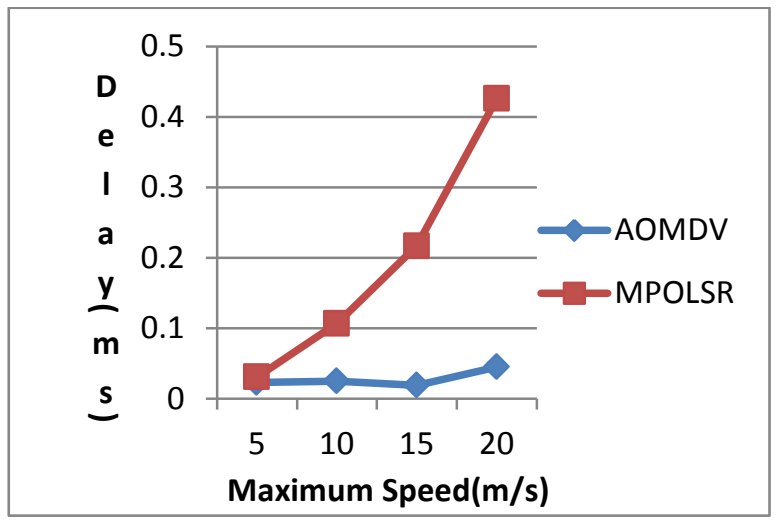

Fig:Comparison of AOMDV \& MPOLSR on the basis of delay at nodes-50, max. Connection-10, pausetime-0 \& by varying speed.

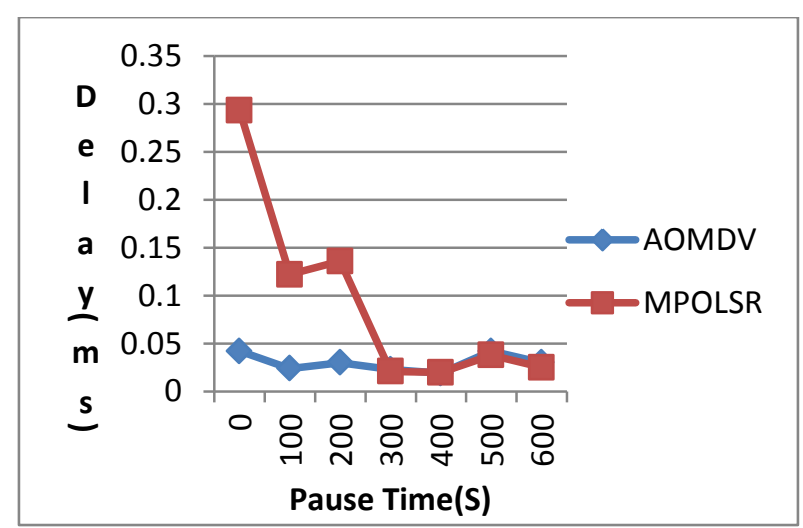

Fig: Comparison of AOMDV \& MPOLSR on the basis of delay at nodes-50, max. Connection-10, max.speed-20 \& by varying pause time. 


\section{REFERENCES}

[1]. M.K. Marina, S.R. Das, On-demand multi path distance vector routing in ad hoc networks, in Proceedings of the Ninth International Conference on Network Protocols, IEEE Computer Society, Washington, DC, USA, 2001, pp. 14-23.

[2]. S.Lee, M. Gerla, Split multipath routing with maximally disjoint paths in ad hoc networks, Helsinki, Finland, 2001, pp. 3201-3205.

[3]. C.Perkins, E. Royer, Ad-hoc on-demand distance vector routing, in: Second IEEE Workshop on Mobile Computing Systems and Applications, 1999, pp. 90-100.

[4]. H. Badis, K.A. Agha, Qolsr multi-path routing for mobile Adhoc networks based on multiple metrics: bandwidth and delay, in: IEEE Vehicular Technology Conference, Los Angeles, CA, USA, 2004, pp.2181-2184.

[5]. M. Kun, Y. Jingdong, R. Zhi, The research and simulation of multipath OLSR for mobile ad hoc network, in: International Symposium on Communications and Information Technologies (ISCIT), 2005, pp.540-543.

[6]. Nasipuri and S. R. Das, "On-demand multipath routing for mobile Adhoc networks," Computer CommunicationsandNetworks, 1999.Proceeding Eight International Conference on, Boston, MA, 1999.

[7]. M. Marina and S. Das, "On-demand Multipath Distance Vector Routing in Ad Hoc Networks", in Proceedings of the International Conference for Network Protocols (ICNP), Riverside, Nov. 2001

[8]. R.Krishan and J.A. Silvester. Choice of allocation granularity in multipath source routing schemes. In IEEE INFOCOM'99, volume 1, pages322-329, IEEE, 1993.

[9].S.J. Lee and M. Gerla. AODV-BR: Backup in ad hoc network. In IEEE WCNC'O0, IEEE, 2000.

[10].A Nasipuri and S.R. Das. On-demand multi-path routing for mobile adhoc networks. In IEEE ICCCN'99, pages 64-70, IEEE, 1999. 